\title{
Erratum: "Green's Function Method in the Problem of Complex Configurations in Fermi Systems with Pairing"

\author{
[Physics of Atomic Nuclei 67, 183-194 (2004)]
}

\section{S. P. Kamerdzhiev and E. V. Litvinova}

In our calculations, numerical errors were made, which led to overestimating some matrix elements of quasiparticle-phonon interaction in the proton subsystem (within the approximations used). Here, we present, in a tabular form, the integrated features of the $E 1$ resonance that were rescaled to $\mathrm{Sn}$ isotopes by using the parameter value of $f_{\mathrm{ex}}=-1.4$, which was taken from the calculations for ${ }^{40} \mathrm{Ca},{ }^{48} \mathrm{Ca}$, and ${ }^{56} \mathrm{Ni}$ [1]. As might have been expected, there are

Integrated features of the isovector $E 1$ resonance in $\mathrm{Sn}$ isotopes

\begin{tabular}{|c|c|c|c|c|c|c|}
\hline Isotope & \multicolumn{2}{|c|}{${ }^{104} \mathrm{Sn}$} & \multicolumn{2}{|c|}{${ }^{120} \mathrm{Sn}$} & \multicolumn{2}{|c|}{${ }^{132} \mathrm{Sn}$} \\
\hline Method & 1 & 2 & 1 & 2 & 1 & 2 \\
\hline EWSR [\%] & 108.4 & 108.3 & 103.6 & 103.3 & 96.1 & 95.8 \\
\hline $\bar{E}[\mathrm{MeV}]$ & 15.0 & 15.2 & 14.4 & 14.6 & 13.2 & 13.4 \\
\hline$\Gamma[\mathrm{MeV}]$ & 4.4 & 1.6 & 5.0 & 2.2 & 4.0 & 2.0 \\
\hline $\begin{array}{l}\sigma_{\text {int }} \\
(0-12 \mathrm{MeV})[\%]\end{array}$ & 20 & 7 & 17 & 11 & 24 & 15 \\
\hline $\begin{array}{l}\sigma_{\text {int }} \\
(0-\bar{E} / 2)[\%]\end{array}$ & 1.3 & 0.17 & 4.0 & 0.37 & 1.4 & 0.24 \\
\hline
\end{tabular}

sizable distinctions (not greater than $50 \%$, as a rule) only for a pygmy dipole resonance. As to Figs. 13 , the behavior of the curves there did not undergo any significant changes, with the exception of what is concerned with a sharp peak at $E \simeq 19 \mathrm{MeV}$ in the region of ${ }^{104} \mathrm{Sn}$ [in a full calculation, the height of this peak decreased to $\sigma(E=19 \mathrm{MeV})=120 \mathrm{mb}$ ].

The corrected results introduce virtually no changes in the conclusions drawn from our study. In particular, the most interesting conclusion that the inclusion of complex configurations is necessary for describing a pygmy resonance remains in force.

In expression (33), the function $G_{1}^{+}(\epsilon)$ was erroneously omitted in the integrand, so that the correct integral must have the form

$$
\int \frac{d \epsilon}{2 \pi i} G_{1}^{+}(\epsilon) M_{1}^{+}(\epsilon) G_{1}^{+}(\epsilon) M_{1}^{+}(\epsilon) G_{1}^{+}(\epsilon) G_{2}^{-}(\epsilon-\omega),
$$

where $G_{1}^{+}(\epsilon)$ and $M_{1}^{+}(\epsilon)$ are the pole parts of the relevant Green's function and mass operator, respectively.

\section{REFERENCES}

1. S. Kamerdzhiev, J. Speth, and G. Tertychny, Nucl. Phys. A 624, 328 (1997).

Translated by A. Isaakyan 